\title{
The differing tempo of growth in bone size, mass, and density in girls is region-specific
}

\author{
Shona Bass, ${ }^{1}$ Pierre D. Delmas, ${ }^{2}$ Georgina Pearce, ${ }^{1}$ Elke Hendrich, ${ }^{1}$ \\ Aaron Tabensky, ${ }^{1}$ and Ego Seeman ${ }^{1}$ \\ ${ }^{1}$ Endocrine Unit and Department of Medicine, Austin and Repatriation Medical Centre, University of Melbourne, \\ Melbourne 3084, Australia \\ ${ }^{2}$ INSERM Research Unit 403, E. Herriot Hospital, cedex 0369437, Lyon, France \\ Address correspondence to: Ego Seeman, Endocrine Department, Austin and Repatriation Medical Centre, Heidelberg, \\ Melbourne 3084, Australia. Phone: 61-3-9496-5489; Fax: 61-3-9496-3365; E-mail: ego@austin.unimelb.edu.au. \\ Shona Bass's present address is: the School of Health Sciences, Deakin University, Burwood, Melbourne 3125, Australia. \\ Received for publication April 12, 1999, and accepted in revised form August 5, 1999.
}

\begin{abstract}
The differing tempo and direction of growth of the periosteal and endocortical surfaces, and the differing tempo of growth of the axial and appendicular skeleton, may predispose to regional deficits in bone size, bone mineral content (BMC), and volumetric bone mineral density (vBMD). These traits were measured during 2 years by dual $x$-ray absorptiometry in 109 girls. By 7 years of age, bone size was approximately $80 \%$ of its maturational peak, and BMC was approximately $40 \%$ of its peak. Before puberty, the legs grew more rapidly than the trunk. During puberty, the growth spurt was truncal. Between 7 and 17 years, femoral and lumbar spine BMC increased by $50-150 \%$ because bone size increased. vBMD increased by $10-30 \%$. Thus, growth builds a bigger, but only moderately denser, skeleton. Regions growing rapidly, or distant from their peak, may be more severely affected by illness than those growing slowly or nearer completion of growth. Depending on the age of exposure to disease, deficits may occur in limb dimensions (prepuberty), spine dimensions (early puberty), or vBMD by interference with mineral accrual (late puberty). As vBMD is independent of age before puberty, the position of an individual's vBMD in the population distribution is established early in life. Bone fragility in old age may have its foundations in growth.
\end{abstract}

J. Clin. Invest. 104:795-804 (1999).

\section{Introduction}

Fractures are a public health problem affecting up to $40 \%$ of women and $15 \%$ of men (1). The bone fragility predisposing to fractures is conferred, in part, by a reduction in areal bone mineral density (aBMD). This deficit is often greatest at the fracture site. For example, women with spine fractures have lower spine than hip aBMD, women with femoral neck fractures have lower femoral neck than spine $\mathrm{aBMD}$, and women with distal radial fractures have greater deficits at the radius than at other sites (2-5).

As all persons lose bone with advancing age, the greater deficit in aBMD at the site of fracture is commonly attributed to excessive bone loss $(3,4)$. However, aBMD in the elderly is a function of the amount of bone gained during growth and the amount of bone lost during aging. The time interval between the attainment of peak aBMD in the second or third decade and the occurrence of vertebral fractures more than 3 decades later probably precludes prospective evaluation of the relative contribution of reduced peak aBMD to the deficit in aBMD in patients with fractures. Nevertheless, some insight has been obtained from studies of the familial resemblance of aBMD in patients with fractures and in their offspring (6-9).

Finding reduced aBMD in the offspring of patients with fractures is consistent with the notion that attainment of a low peak aBMD during growth is likely to account partly for the deficit in aBMD in these patients (6-9). As the deficit in aBMD in offspring (relative to their age-matched peers) is about half the deficit in their parents (relative to their age-matched peers), and parents and offspring share half their genes, the low peak aBMD may be a sufficient explanation for the reduced aBMD; bone loss, in excess of that seen in the population, need not be invoked.

Furthermore, the greater deficits in aBMD at the fracture site in patients with osteoporosis may be due to regional deficits in peak bone size, aBMD, or both (rather than excessive bone loss) because daughters of women with spine fractures have greater deficits in aBMD at the spine than femur (6); daughters of women with femoral neck fractures have deficits in aBMD confined to the femoral neck and midshaft (7); women and men with spine fractures have reduced vertebral body size, but not femoral neck size (10-12); and men with hip fractures have reduced femoral neck size but normal vertebral body size (12). Thus, factors operating during the first 15 years of life may determine whether an individual has a smaller or larger bone with lower or higher aBMD at one site but not another. Hence, studies of skeletal growth may advance our understanding of the pathogenesis of bone fragility in old age.

The purpose of this study was to describe the age- and pubertal stage-specific tempo of growth in bone size and mineral content of the axial and appendicular skeleton. On the basis of the observations made in this study, we propose that the differing tempo of growth of size and mass of a region, and the differing tempo of growth of one region relative to another, predispose to region-specific 
deficits in bone size, mass, and density, which in turn contribute to the differing types of fractures in old age.

\section{Methods}

Subjects. One hundred and nine volunteers from the Ivanhoe Girls Grammar School were recruited after written permission was obtained from the girls, their parents, and the school principal. All the subjects were Caucasian, healthy, and with no diseases, exposure to contraceptives, or drugs known to affect bone. Data were collected at 6 monthly intervals for 2 years. The study was approved by the Austin and Repatriation Medical Centre Ethics Committee.

Bone densitometry, anthropometry, and bone age. Bone mass was measured using dual x-ray absorptiometry (Lunar DPX-L, version 1.3z;Lunar Corp., Madison, Wisconsin, USA) (13). Results were expressed as bone mineral content (BMC; $\mathrm{g}$ ) and aBMD (BMC/projected area of the region scanned; $\left.\mathrm{g} / \mathrm{cm}^{2}\right)$. Regional $\mathrm{BMC}$ was determined by using the "region of interest" option from the total body scan. Spine BMC refers to the spine including third cervical to fifth lumbar vertebrae. The coefficient of variation (CV) was $2-4 \%$.

$\mathrm{BMC}$ within a $4 \mathrm{~cm}^{2}$ projected area centered at the midshaft of the femur was determined using the pediatric antero-posterior spine program. The ruler function was used to determine the periosteal and endocortical diameters. The $\mathrm{CV}$ was $1.5 \%$. Femoral midshaft volumetric bone mineral density (vBMD) was calculated as BMC divided by the total shaft volume (i.e., cortical plus medullary volumes, assuming these to be cylindrical). True BMD of the femoral cortex (i.e., the cortical bone "inside" periosteum and "outside" the endocortical envelope) was calculated as BMC/cortical volume (total shaft minus medullary volumes). The true BMD (mineral mass per unit volume of mineralized bone matrix itself) is about $2.05 \mathrm{~g} / \mathrm{cm}^{3}$ by chemical methods (14). Accuracy errors may occur in the derivation of true BMD because intracortical canals and intracanalicular volumes are ignored (15), and the estimate assumes that the midshaft of the femur is a cylinder. All the other measures of "density," namely, BMC, aBMD, vBMD, are "apparent" density measurements of the whole bone (marrow space included), although not prefaced in this article by the word "apparent" (16). Periosteal and endocortical diameters at the midpoint of the third metacarpal were measured using radiogrametry. vBMD of the third lumbar vertebra (L3) was calculated as BMC/vertebral body volume, where volume $=\operatorname{area}^{3 / 2}(17)$. The CV was 0.9-2.9\%. The vBMD calculation is likely to be an overestimate (18), as rectilinear postero-anterior scanning includes the mass of the posterior processes; the growth in size of these structures is not taken into account by the Carter method of deriving vBMD (17).

Standing height $(\mathrm{cm})$ was measured using a Holtain stadiometer. Femur length (the distance from the inferior border of the lateral epicondyle to the superior border of the greater trochanter) and tibia, humerus, and ulna lengths $(\mathrm{mm})$ were measured using a Harpenden anthropometer. Bone age was determined at baseline and 18 months using the Greulich and Pyle method.
Bone age was used because any delay in puberty will result in a reduced bone mass and size relative to chronological age but not necessarily relative to bone age. Pubertal status was assessed using Tanner breast staging $(1=$ prepubertal, $2-4$ = peripubertal, postpubertal $=$ after menarche $)$.

Biochemical measurements. A 12-hour overnight urine sample was collected before testing. Serum from a fasting blood sample was stored at $-40^{\circ} \mathrm{C}$. Osteocalcin was measured with a human specific immunoradiometric assay (OC, ng/mL; ELSA-OSTEO, Cis Biointernational, Salcay, France) (19). Bone-specific alkaline phosphatase was measured using an immunoradiometric assay (BSAP, ng/mL; Ostare; Hybritech Inc., San Diego, California, USA) (20). Collagen propeptide of type 1 collagen was measured with a 2-site ELISA (PICP, ng/mL; Procollagen-C; Metra Biosystems, Mountain View, California, USA) (21). Bone resorption was assessed by measuring urinary type $1 \mathrm{C}$-telopeptide breakdown products with an ELISA $(\mu \mathrm{L} / \mathrm{mmol}$, CrossLaps; Osteometer A/S, Herlev, Denmark) (22). The intra- and interassay CVs were $7-10 \%$ and $7-13 \%$, respectively.

Serum estradiol (E2, pmol/L) and testosterone (nmol/L) were assayed using an ACS:180 Automated Chemiluminescent System (Ciba-Corning Diagnostics Corp., Medfield, Massachusetts, USA). The E2 and testosterone assays had sensitivities of $55 \mathrm{pmol} / \mathrm{L}$ and $0.5 \mathrm{nmol} / \mathrm{L}$, respectively, and interassay $\mathrm{CV}$ of $8 \%$ and $7 \%$, respectively. Commercial RIA kits were used to measure growth hormone $(\mathrm{GH}, \mathrm{mIU} / \mathrm{L}$; Spectria, Human Growth Hormone, Orion Diagnostica, Espoo, Finland), dehydro-epiandrosterone sulfate (DHEAS, $\mathrm{ng} / \mathrm{mL}$; DHEAS Direct, Biotecx, Houston, Texas, USA), and androstenedione ( $\mathrm{ng} / \mathrm{mL}$; Direct Androstenedione, Diagnostics Biochem Canada Inc., Ontario, Canada). The interassay CVs were 4\%, 9\%, and 14\%, respectively. IGF-1 (ng/mL) was measured by RIA using anti-human IGF-1 antibodies raised in rabbits (GroPep, Adelaide, Australia) and had an interassay CV of 5\%.

Statistical analyses. The data are presented in absolute terms, percentage terms, and as a rate of change (centimeters per month and grams per month). To define the relative patterns of growth in bone size and mass, each trait was expressed as a percentage of the predicted adult peak derived from data in the girls with regular menstrual cycles for 2-4 years. For the mixed crosssectional and longitudinal analyses, ANOVA followed by the Fisher method of multiple comparisons was used to compare mean values at each bone age interval. A significance level of $P<0.05$ was used for the ANOVA, and 95\% confidence level was used for the Fisher comparisons. To determine whether variances increased as puberty advanced, bone age-adjusted residuals for BMC and bone size were plotted against bone age. Levene's test for variances was used to determine significance.

In the calculation of the percentage deficit relative to peak BMC and bone size, we assumed that peak young adult values were achieved at the age of 16 years (23). The values were derived using data in 36 girls with regular menstrual cycles for at least 4 years, age $16.5 \pm 0.2$ years, height $163.9 \pm 1.0 \mathrm{~cm}$, total body BMC 2,460 \pm 67 $\mathrm{g}$, and spine BMC $239 \pm 9 \mathrm{~g}$; measurements comparable 
with those found in 18 women age $24.7 \pm 0.5$ years, height $164.2 \pm 1.5 \mathrm{~cm}$, total body BMC 2,599 $\pm 93 \mathrm{~g}$, and spine BMC $250 \pm 10 \mathrm{~g}$. To compare the growthrelated increases in $\mathrm{BMC}$ and $\mathrm{VBMD}$, we regressed each trait on age and compared the ratios of the slope coefficient/residual standard error and the increase expressed in standard deviation scores.

A mixed cross-sectional longitudinal design was used, as this gives an accurate age-specific mean for the trait being studied (24). There was continuity in the crosssectional and longitudinal data as the absolute values and variance for anthropometric and bone mass measurements did not differ between the subjects tested for the first time at a given age and subjects now at the same age having their first measurement 2 years earlier.

The associations between bone size and mass with sex hormones, GH and IGF-1, were determined during the accelerating and the decelerating phases of growth. Baseline periosteal and endocortical diameters were correlated with hormone measurements. Significantly correlated results were entered into a stepwise regression to identify independent predictors of each trait. All correlations were adjusted for bone age.

\section{Results}

At the onset of the study, the participants had a mean bone age of 12 years (range, 6.5-17) and mean chronological age of 12 years (range, 6.9-16.6). Bone age and chronological age did not differ (mean difference $=-0.03$ \pm 0.1 years). Thirty-six girls were prepubertal, with a bone age of 9.3 years (range, 6.3-12) and a chronological age of 9.4 years (range, 6.9-11.9); 26 girls were peripubertal, with a bone age 11.8 years (range, 9.6-15) and a chronological age of 12.0 years (range, 10.1-15.1); 36 girls were postpubertal, with a bone age of 14.9 years (range, 12-17) and a chronological age of 14.6 years (range, 11.4-16.6). Five girls refused assessment of pubertal status.

Growth in bone length and bone mass. Table 1 shows the absolute values for anthropometric and bone mass measurements. Figure 1 shows the results expressed as a percentage of the predicted adult value versus bone age. The shaded region in Figure 1 represents pubertal growth from Tanner stage 2 to menarche. In prepubertal girls with a bone age of 7 years, height, sitting height, and region lengths were approximately $80 \%$ of the predicted adult value; total and regional BMC were approximately $40 \%$ of the predicted adult value. At menarche
Figure 1

Height, regional lengths, and total body and regional BMC expressed as a percentage of the predicted young adult peak value versus bone age. The shaded area represents the pubertal growth period (Tanner stage 2 to menarche).
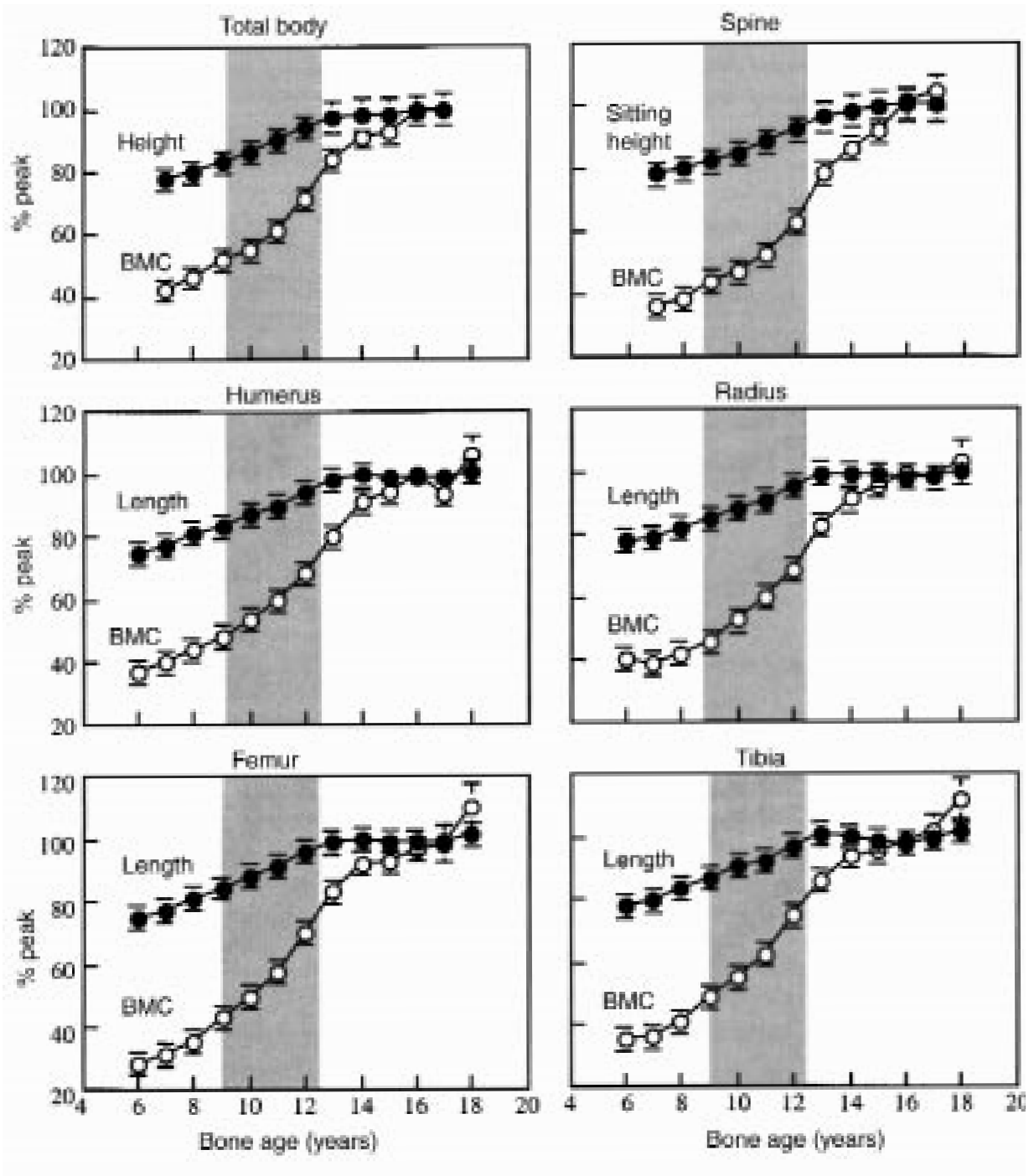
Figure 2

The bone-age adjusted residuals for spine and leg BMC increased with advancing age, whereas the bone age adjusted residuals for sitting height and leg length did not. Prepubertal (open circles), peripubertal (filled circles), and postpubertal (filled diamond symbols).
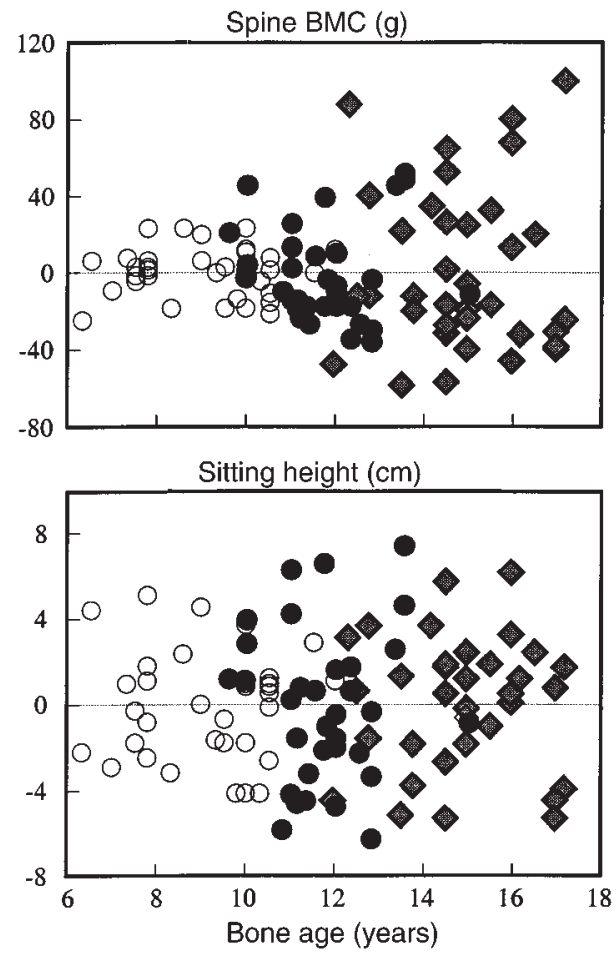

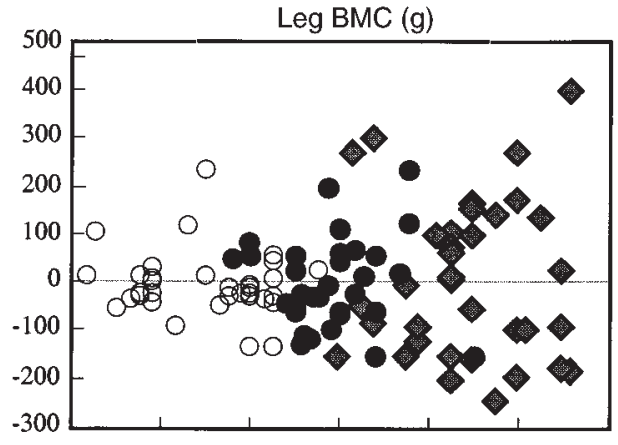

Leg length $(\mathrm{cm})$

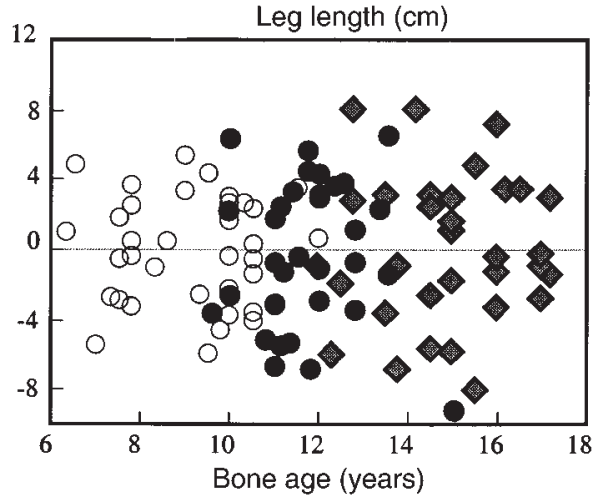

$(12.7 \pm 0.1$ years), bone lengths were within $3 \%$ of their adult peak; total and regional BMC were $15-20 \%$ below their predicted peak.

Figure 2 shows that the variance in the age-adjusted $\mathrm{BMC}$ residuals increased with advancing bone age, whereas age-adjusted residuals for bone length did not. The pre-, peri-, and postpubertal BMC residuals $\left(\mathrm{g}^{2}\right)$ increased, being, respectively, $174 \pm 34.6,626 \pm 139$, and $1,838 \pm 381$ (spine, $P<0.001$ ), and 4,777 $\pm 1729,8,775 \pm$ 2,229 , and $27,237 \pm 5,539$ (legs, $P<0.05$ to 0.001 ). The respective pre-, peri-, and postpubertal length residuals $\left(\mathrm{cm}^{2}\right)$ did not increase significantly and were $6.1 \pm 1.3$, $12.9 \pm 2.7,9.7 \pm 1.9$ (sitting height), and 9.6 $\pm 1.7,17.5 \pm$ $10.6,17.3 \pm 3.7$ (leg length).
Figure 3 shows the prospectively derived rates of total and regional growth in bone size and BMC versus bone age. Growth velocity of sitting height slowed before puberty, accelerated, and then decelerated at approximately 12 years. Growth velocity of the legs was more rapid than that of sitting height before puberty. In contrast to the spine, the legs showed no significant acceleratory phase before decelerating rapidly at approximately 11 years. There was no detectable increase in sitting height after 14 years of age or in leg length after 16 years of age.

At approximately 7 years, mineral accrual at the legs was higher than at the spine $(5.1 \pm 0.6$ versus $0.8 \pm 0.5$ $\mathrm{g} /$ month). At approximately 12 years, mineral accrual was higher in the legs than spine $(10.8 \pm 0.8$ versus $3.8 \pm$

Table 1

Height, sitting height, appendicular segment lengths, total and regional bone mineral content in 109 girls aged 7 to 17 years (mean \pm SEM)

\begin{tabular}{|c|c|c|c|c|c|c|c|c|c|c|c|}
\hline $\begin{array}{l}\text { Bone age (years) } \\
n\end{array}$ & $\begin{array}{c}7 \\
(23)\end{array}$ & $\begin{array}{c}8 \\
(23)\end{array}$ & $\begin{array}{c}9 \\
(27)\end{array}$ & $\begin{array}{c}10 \\
(72)\end{array}$ & $\begin{array}{c}11 \\
(53)\end{array}$ & $\begin{array}{c}12 \\
(65)\end{array}$ & $\begin{array}{c}13 \\
(51)\end{array}$ & $\begin{array}{c}14 \\
(36)\end{array}$ & $\begin{array}{c}15 \\
(57)\end{array}$ & $\begin{array}{c}16 \\
(34)\end{array}$ & $\begin{array}{c}17 \\
(34)\end{array}$ \\
\hline \multicolumn{12}{|c|}{ Bone length } \\
\hline Height $(\mathrm{cm})$ & $127.9 \pm 1.1$ & $132.4 \pm 0.9$ & $136.8 \pm 0.9$ & $142.8 \pm 0.7$ & $148.0 \pm 0.8$ & $154.7 \pm 0.8$ & $160.2 \pm 1.0$ & $162.2 \pm 1.1$ & $162.3 \pm 0.9$ & $163.8 \pm 1.2$ & $164.6 \pm 0.9$ \\
\hline Sitting height $(\mathrm{cm})$ & $68.3 \pm 0.6$ & $70.0 \pm 0.4$ & $71.7 \pm 0.5$ & $74.7 \pm 0.3$ & $77.7 \pm 0.5$ & $80.6 \pm 0.4$ & $84.1 \pm 0.5$ & $86.0 \pm 0.6$ & $87.0 \pm 0.4$ & $88.1 \pm 0.5$ & $87.3 \pm 0.5$ \\
\hline Humerus (mm) & $268 \pm 2$ & $281 \pm 2$ & $289 \pm 3$ & $303 \pm 2$ & $312 \pm 2$ & $329 \pm 2$ & $343 \pm 3$ & $347 \pm 3$ & $344 \pm 2$ & $344 \pm 3$ & $345 \pm 3$ \\
\hline Radius-ulna (mm) & $199 \pm 2$ & $206 \pm 2$ & $214 \pm 2$ & $222 \pm 1$ & $229 \pm 1$ & $240 \pm 1$ & $249 \pm 2$ & $250 \pm 2$ & $251 \pm 2$ & $247 \pm 3$ & $249 \pm 2$ \\
\hline Tibia (mm) & $288 \pm 3$ & $301 \pm 3$ & $314 \pm 3$ & $327 \pm 2$ & $335 \pm 3$ & $352 \pm 3$ & $365 \pm 3$ & $362 \pm 4$ & $357 \pm 3$ & $355 \pm 4$ & $362 \pm 2$ \\
\hline \multicolumn{12}{|c|}{ Bone mineral content } \\
\hline Total Body (g) & $1011 \pm 24$ & $1101 \pm 35$ & $1251 \pm 29$ & $1360 \pm 21$ & $1503 \pm 31$ & $1729 \pm 34$ & $2034 \pm 46$ & $2217 \pm 60$ & $2294 \pm 39$ & $2435 \pm 31$ & $2506 \pm 82$ \\
\hline Spine (g) & $84 \pm 3$ & $89 \pm 3$ & $99 \pm 3$ & $111 \pm 3$ & $127 \pm 4$ & $146 \pm 34$ & $184 \pm 6$ & $205 \pm 7$ & $219 \pm 5$ & $239 \pm 10$ & $241 \pm 9$ \\
\hline Humerus (g) & $24.8 \pm 0.7$ & $27.9 \pm 1.0$ & $30.2 \pm 0.7$ & $33.7 \pm 0.6$ & $37.7 \pm 1.0$ & $43.3 \pm 1.0$ & $50.5 \pm 1.3$ & $57.4 \pm 1.7$ & $59.6 \pm 1.4$ & $62.8 \pm 2.1$ & $61.6 \pm 2.2$ \\
\hline Radius-ulna (g) & $19.6 \pm 0.6$ & $21.2 \pm 0.8$ & $23.2 \pm 0.7$ & $26.7 \pm 0.5$ & $30.6 \pm 0.8$ & $34.8 \pm 0.8$ & $41.9 \pm 1.0$ & $46.5 \pm 1.4$ & $48.6 \pm 1.2$ & $50.6 \pm 1.7$ & $51.0 \pm 1.7$ \\
\hline Femur $(\mathrm{g})$ & $70.7 \pm 2.1$ & $81.8 \pm 3.3$ & $99.2 \pm 4.2$ & $113.4 \pm 2.9$ & $132.0 \pm 4.2$ & $161.4 \pm 4.3$ & $192.2 \pm 5.6$ & $211.6 \pm 7.4$ & $214.2 \pm 6.2$ & $222.1 \pm 7.7$ & $235.8 \pm 10$ \\
\hline Tibia-fibula (g) & $58.1 \pm 1.9$ & $65.3 \pm 2.7$ & $78.2 \pm 2.7$ & $88.3 \pm 2.1$ & $99.8 \pm 2.9$ & $119.7 \pm 3.1$ & $136.7 \pm 3.4$ & $149.5 \pm 5.1$ & $151.8 \pm 3.9$ & $156.9 \pm 5.4$ & $167.8 \pm 7.1$ \\
\hline
\end{tabular}


$0.6 \mathrm{~g} /$ month, respectively) despite growth in leg and spine lengths being similar $(\sim 0.3 \mathrm{~cm} / \mathrm{month})$. Deceleration in mineral accrual occurred 1 year later than deceleration in bone length at both sites. Bone mass accrual continued at the spine and legs until 16 years of age.

Between 7 and 17 years, 1,491 g of mineral was accrued: $42 \%$ (630 g) in the legs; $25 \%$ (376 g) in the ribs, sternum, pectoral, and pelvic girdles; $12 \%(176 \mathrm{~g})$ in the arms; $11 \%$ $(157 \mathrm{~g})$ in the skull; and 10\% (156 g) in the spine. Between the $7-11$ years (prepuberty), 11-14 years (puberty), and 14-17 years (postpuberty), the increases in spine BMC were 38, 72, and $46 \mathrm{~g}$, respectively. The corresponding increases in leg BMC were 240, 241, and $149 \mathrm{~g}$.

Growth in BMC and $v B M D$. Midshaft metacarpal and femoral cortical widths were approximately $70 \%$ of the predicted adult peak in prepubertal girls (Tanner stage 1). As shown in Figure 4, periosteal diameter increased at both sites with little change in endocortical diameter. Thus, cortical width increased, particularly when endocortical diameter contracted at Tanner stage 3 at the metacarpal, and after menarche at the femur. Of final cortical width, endocortical contraction contributed $13 \%$ at the metacarpal and $7 \%$ at the femur.

Bone mass measurements were available for the femur, not metacarpal. As shown in Figure 4, as periosteal and endocortical diameters of the femoral midshaft increased comparably, cortical width remained constant between Tanner stage 4 and menarche. Despite this, femoral midshaft vBMD increased, presumably because true BMD of the cortex increased (lighter shaded column). After menarche, periosteal diameter increased and endocortical (medullary) diameter decreased resulting in an increase in cortical width (Figure 4, darkly shaded column). Despite the increasing femoral midshaft cortical width, vBMD remained unchanged, perhaps because true BMD of the cortex decreased.

As shown in Figure 5, BMC at the femoral midshaft increased across age by $50 \%$, or $3.4 \mathrm{SD}$. This was 4 times the increase of $0.86 \mathrm{SD}$ in femoral midshaft vBMD. L3 BMC increased across age by $150 \%$; a 3.7 SD increase, 1.5 times the increase of 2.6 SD in vBMD.

Biochemical and hormonal measurements. Biochemical measures of bone remodeling and serum E2 increased, reached a peak at bone age approximately 12 years (Tanner stage 4) and then decreased at menarche at a serum E2 of 200-400 pmol/mL (Table 2; Figures 6 and 7). Change in total bone mass (grams per month) correlated with alkaline phosphatase $(r=0.20)$, osteocalcin $(r=$ $0.38)$, P1CP $(r=0.32)$, and CrossLaps $(r=0.24)$ (all $P<$ $0.02)$. Serum E2 accounted for $28 \%$ of the variance in leg BMC accrual in the accelerating phase of growth, 19\% of the variance in the spine BMC accrual, and $49 \%$ of the variance in cortical width in the decelerating phase of growth (both, $P<0.05$ ). IGF-1 accounted for $21 \%$ of the variance in the spine BMC in the accelerating phase of growth and for $14 \%$ of the variance in endocortical diameter (both $P<0.03$ ). Bone age was the only independent predictor of leg bone mass accrual accounting for $35 \%$ of the variance $(P<0.005)$.

\section{Discussion}

We report that $\mathrm{BMC}$ and $\mathrm{BBMD}$ increased during growth. However, the changes were trait, surface, and region specific: the age of onset, peak growth velocity, and completion of growth of a region's external dimensions preceded the mineral accrued within its periosteal envelope. Before puberty, appendicular growth was more rapid than axial growth. In early puberty, appendicular growth remained constant with no acceleration, whereas axial growth accelerated. Growth of both regions then decelerated in late puberty. Cortical bone width increased due to periosteal expansion followed by endocortical contraction. vBMD of the midshaft of the femur and spine increased.

Growth in size and mass within a region. The differing tempo of growth of bone size and mass within a region may contribute to deficits in bone size, BMC, and vBMD found in
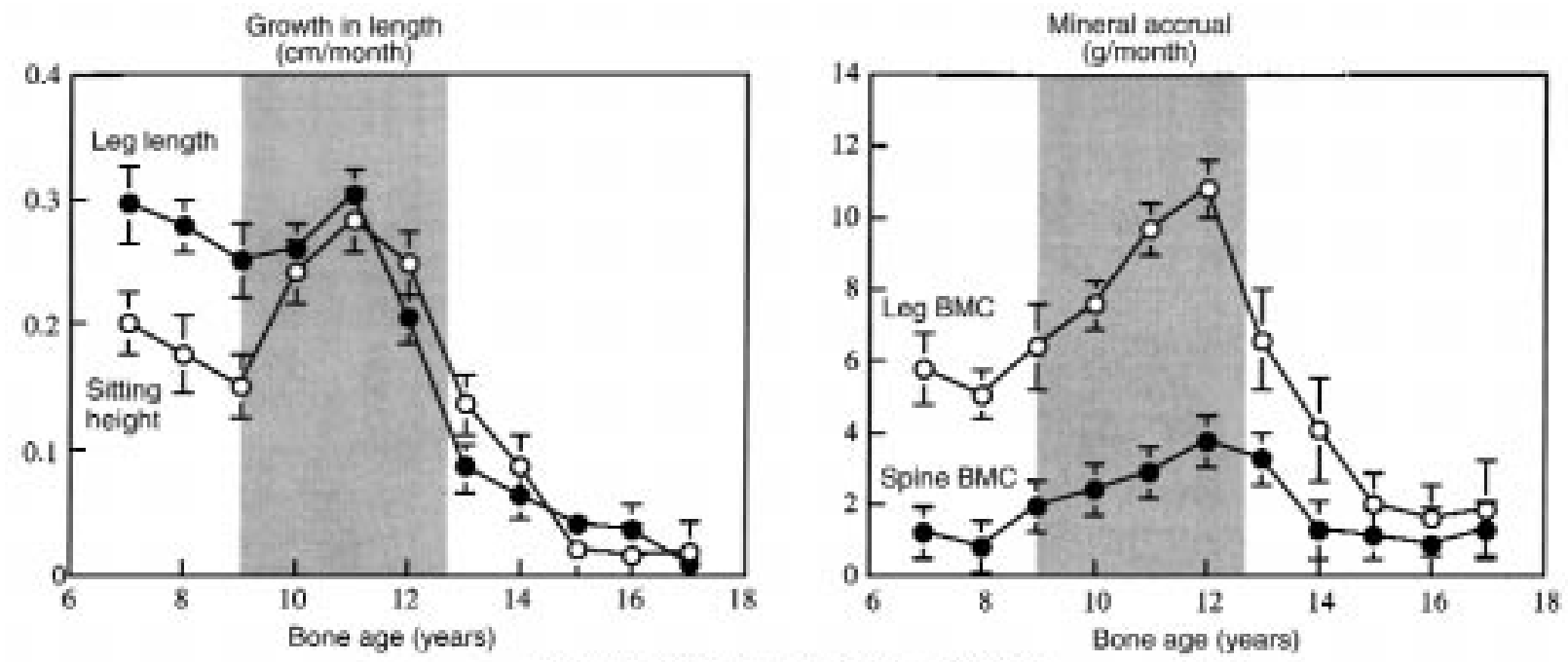

$n=20,22,19,64,40,50,34,24,41,20,26$

Figure 3

Rates of growth in regional bone length (centimeters per month) and bone mass (grams per month) versus bone age (mean \pm SEM). The shaded area represents the pubertal growth period (Tanner stage 2 to menarche). 
Figure 4

Midshaft metacarpal and femoral dimensions and femoral midshaft vBMD and cortical true BMD as a function of pubertal status.
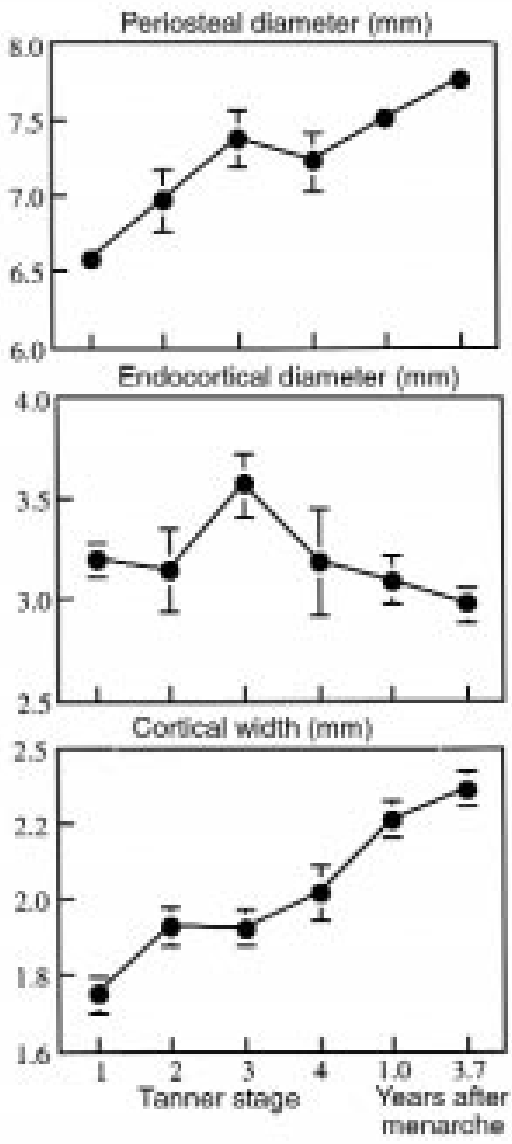

$m=54, \quad 13, \quad 17, \quad 11, \quad 23, \quad 40$ Man Buns A pe (ars) $9.4112,12.112 .4,13.6,16.2$
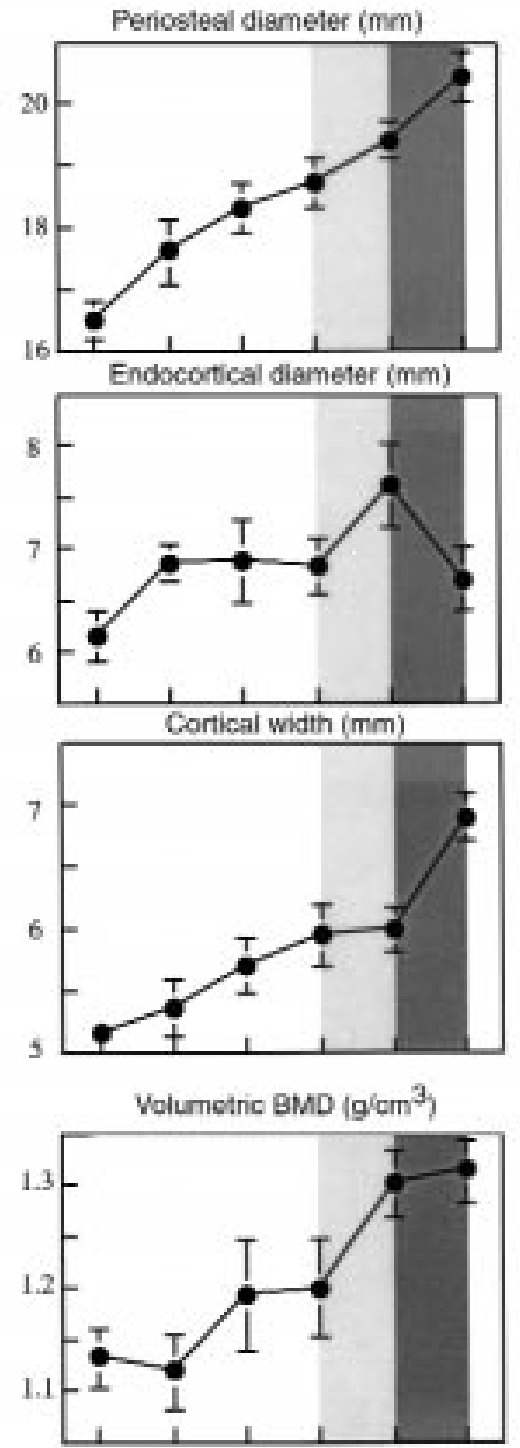

patients with fractures (12). As a region's size is nearer its peak than its mass (25), illness interrupting growth may result in a greater deficit in peak bone mass than peak bone size, leading to a reduced vBMD. For example, in the growing rat, ovariectomy results in excessive appendicular growth (due to failed epiphyseal closure), but similar mineral accrual relative to sham operated controls. Thus, the increase in mass is less relative to the increase in size, resulting in reduced vBMD (26). Likewise, GH or IGF-1 administration to rats results in a greater relative increase in size than mass so that vBMD decreases (27). The earlier growth in size than mass in childhood may increase bone fragility and account for the increased forearm fractures observed at around 12-13 years of age (28).

Growth between regions: axial versus appendicular. Likewise, the differing tempo of growth between regions may predispose to deficits in size, mass, or vBMD at one region but not another. Rapidly growing regions, or regions further from their peak, may be more severely affected by illness than are slowly growing regions or regions that have completed their growth. Before puberty, growth of the legs was more rapid than growth of the spine; $40 \%$ of the $630 \mathrm{~g}$ of mineral to be accrued in the legs was accrued by 11 years of age, whereas only $24 \%$ of spine mineral mass had been accrued. At puberty, growth of the spine accelerated while growth of the legs slowed without a detectable acceleration phase. Hypogonadism or delayed puberty produces increased leg length due to failed epiphyseal closure but reduced trunk length due to failed pubertal acceleration of trunk growth (29).

$\checkmark B M D$ : the result of the relative growth of bone size and the amount of bone accrued within its periosteal envelope. vBMD, 
Figure 5

$\mathrm{BMC}$ and $\mathrm{vBMD}$ at the femoral midshaft and third lumbar vertebra versus bone age.
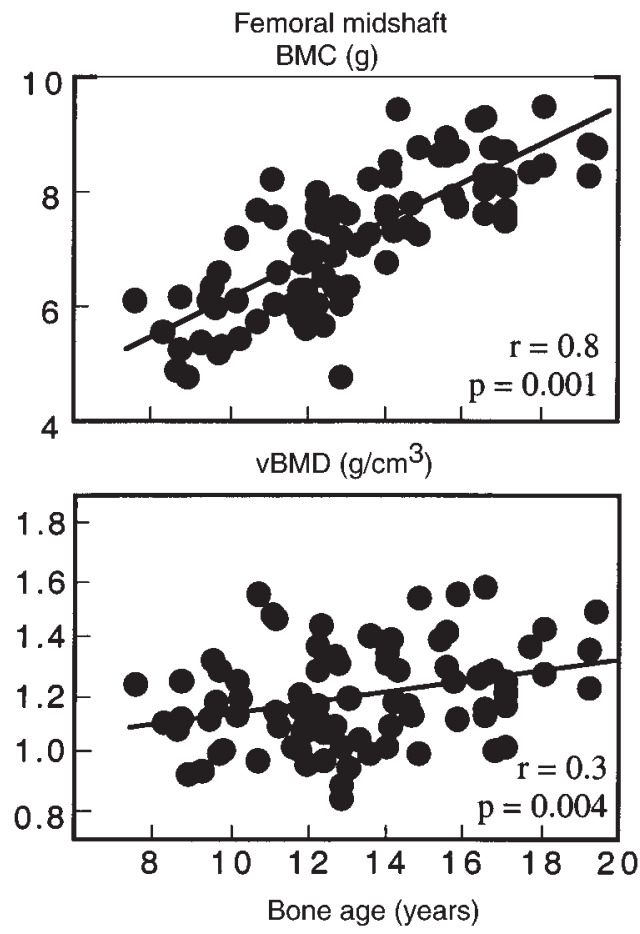

Third lumbar vertebra $\mathrm{BMC}(\mathrm{g})$
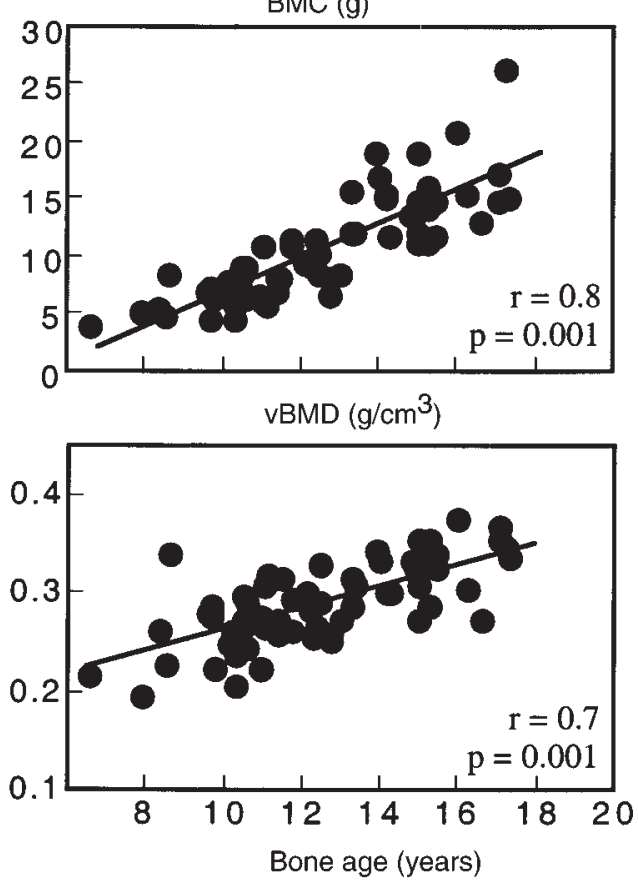

the mass per unit volume of bone, is determined by the relative growth of the external dimensions of the bone and the mineral mass accrued within it (16). vBMD of the femoral midshaft remained relatively constant in early puberty (Tanner 1-4; Figure 4). Similarly, constancy of vBMD of the femoral shaft, radial shaft, and vertebral body has been has been reported by several investigators $(30-34)$. The increase in $\mathrm{VBMD}$ at the spine reported here is likely to be an overestimate because of inclusion of the posterior processes in the measurement (see Methods).

This constancy of vBMD during the prepubertal years is not widely recognized because most studies of growth report BMC or aBMD, size-dependent expressions of "density" that increase with age largely because bone size increases $(16,35-37)$. This constancy of vBMD before puberty suggests that the position of an individual's peak vBMD relative to other individuals in the population distribution is likely to be partly determined during early growth. That is, whether a person's vBMD is at the 5th, 50 th, or 95 th percentile may be partly determined early in life by genetic factors coregulating the relative growth of bone size and mass. vBMD "tracks" because the increase in size during growth is matched by a proportional increase in mass within it, at least before puberty. In morphological terms, the increase in femoral midshaft size was matched by a proportional increase in the cortical width of the enlarging bone. In the spine, vBMD remains constant because the increasing vertebral body size is matched by an increase in the thickness of the existing trabeculae within it $(38,39)$.

By contrast, vBMD increased during late puberty and after menarche. For vBMD to increase - for there to be more bone in the growing bone - the increase in mineral mass within the periosteal envelope must be relatively greater than the increase in the external bone size. In morphological terms, vBMD increased at the femoral midshaft because cortical thickness increased by periosteal expansion, slower endocortical expansion, followed by endocortical contraction after menarche (40). (If both surfaces expand at the same rate, the enlarging bone will not have a thicker cortex. If the endocortical surface expands more rapidly than the

Table 2

GH, IGF-1, serum estradiol, testosterone, androstenedione and, DHEAS according to Tanner stage (mean \pm SEM).

\begin{tabular}{|c|c|c|c|c|c|c|}
\hline & \multicolumn{4}{|c|}{ Tanner stage } & \multicolumn{2}{|c|}{ Years after menarche } \\
\hline & $\begin{array}{c}1 \\
(n=45)\end{array}$ & $\begin{array}{c}2 \\
(n=31)\end{array}$ & $\begin{array}{c}3 \\
(n=42)\end{array}$ & $\begin{array}{c}4 \\
(n=42)\end{array}$ & $\begin{array}{c}1.0 \pm 0.1 \\
(n=75)\end{array}$ & $\begin{array}{l}3.7 \pm 0.1 \\
(n=118)\end{array}$ \\
\hline & \multicolumn{6}{|c|}{ Concentration (mean \pm SEM) } \\
\hline $\mathrm{mlU} / \mathrm{L})$ & $40 \pm 28$ & $18 \pm 3$ & $18 \pm 2$ & $24 \pm 3$ & $22 \pm 2$ & $17 \pm 1$ \\
\hline$(\mathrm{ng} / \mathrm{mL})$ & $144 \pm 7$ & $197 \pm 81$ & $220 \pm 12$ & $301 \pm 14$ & $313 \pm 8$ & $292 \pm 6$ \\
\hline diol (pmol/L) & $87 \pm 7$ & $159 \pm 22$ & $159 \pm 13$ & $195 \pm 18$ & $372 \pm 31$ & $395 \pm 21$ \\
\hline sterone (nmol/L) & $0.80 \pm .04$ & $0.83 \pm 0.05$ & $1.04 \pm 0.06$ & $1.35 \pm 0.10$ & $1.46 \pm .06$ & $1.66 \pm 0.06$ \\
\hline ostenedione $(\mathrm{ng} / \mathrm{mL})$ & $2.0 \pm 0.1$ & $2.8 \pm 0.2$ & $3.3 \pm 0.2$ & $4.1 \pm 0.3$ & $4.8 \pm 0.2$ & $5.9 \pm 0.2$ \\
\hline $\mathrm{AS}(\mathrm{ng} / \mathrm{mL})$ & $752 \pm 74$ & $825 \pm 117$ & $1013 \pm 121$ & $1339 \pm 148$ & $1799 \pm 135$ & $2519 \pm 168$ \\
\hline
\end{tabular}



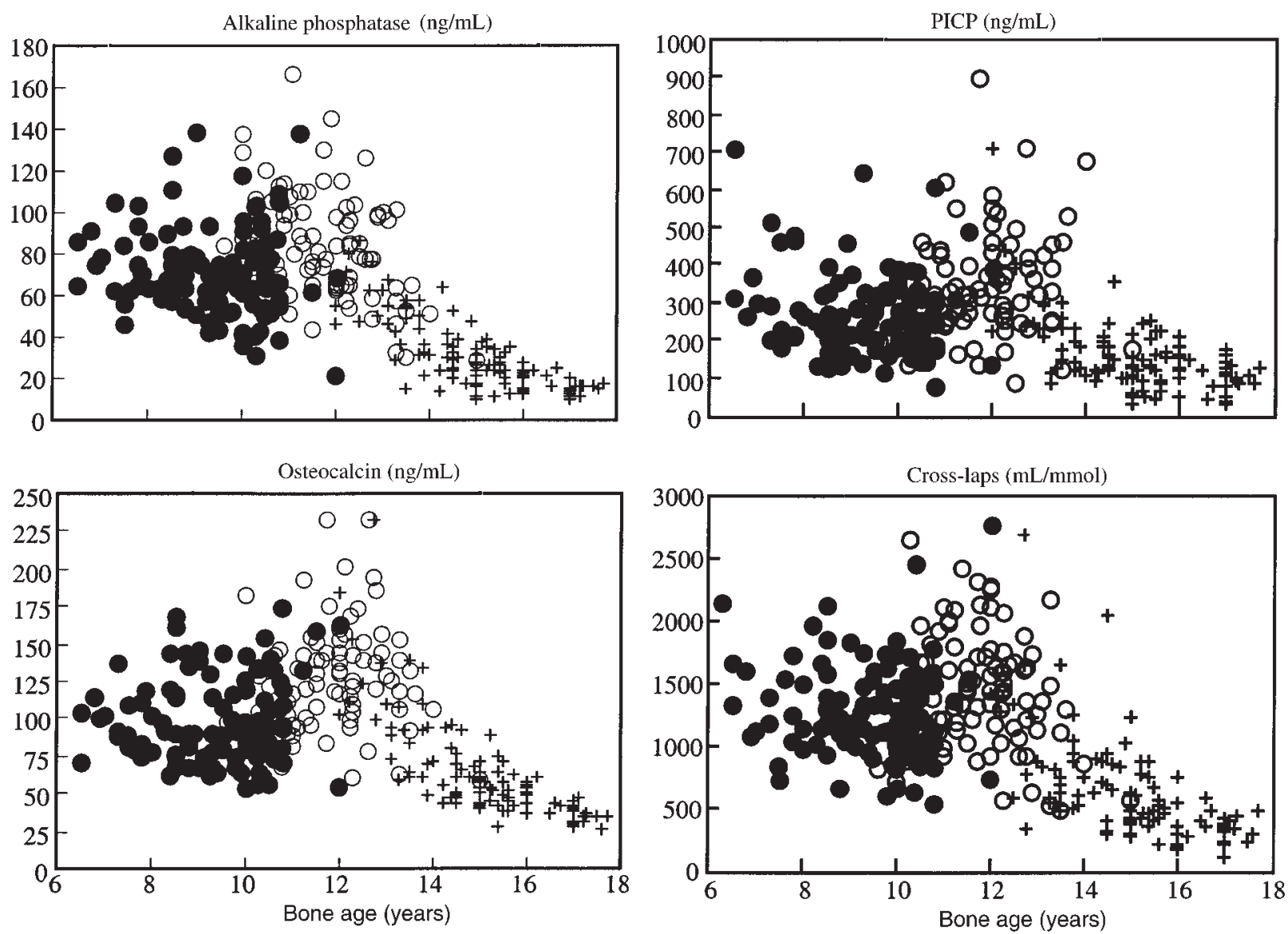

Figure 6

Serum bone specific alkaline phosphatase, osteocalcin, collagen propeptide of type I collagen (PICP), and urinary type I C-telopeptide breakdown products (CrossLaps) versus bone age. Prepubertal (filled circles), peripubertal (open circles), and postpubertal (crosses).

expanding periosteum, cortical thickness will decrease.) vBMD of the vertebral body increases during puberty due to continued, and perhaps accelerated, thickening of trabeculae $(32,34,39)$. The numbers of trabeculae, determined early in life at the growth plate, remain constant from early life to adulthood (39).

The increase in vBMD of the femoral midshaft may have also been partly due to an increase in the true density of the cortical shell at menarche because this increase in vBMD of the shaft occurred when cortical width did not change, whereas true BMD increased (Figure 4). After menarche, endocortical contraction occurred. Despite this, vBMD remained constant because true BMD decreased, perhaps because secondary mineralization of the newly accumulated matrix had not yet occurred. These inferences will require verification using bone histomorphometry given that the calculation of true BMD assumes that the midshaft of the femur is a cylinder.

Although vBMD increased in late puberty, it is likely that $\mathrm{VBMD}$ increased more in some individuals than others. The variance in bone size did not increase, suggesting that bone size may "track," i.e., individuals may gain a similar proportion of their starting value during growth (41). However, the variance in BMC increased during puberty and after menarche (Figure 2), suggest- ing that BMC may not track; some individuals may accrue a greater "dose" of sex hormone-dependent bone mass per unit bone volume, whereas others accrue less bone mass per unit bone volume. In morphological terms, for a given bone size, some individuals may gain a thicker cortex than others by less expansion of the endocortical surface before puberty by less resorptive modeling or by more endocortical apposition during puberty than others. Persons with higher spine vBMD may have formed greater trabeculae numbers at the growth plate during early development or may have increased trabecular thickness during pre- and peripubertal development (more than others with the same starting bone size). The magnitude of the population variance of each of these structural components of bone need to be defined at each age and for both genders. Then, the genetic and environmental components of the variance can be studied $(42,43)$.

In conclusion, the skeleton is not a single functioning entity; the effect of growth, aging, disease, and treatment vary according to whether a region is axial or appendicular, cortical or trabecular, and whether the surface is periosteal or endosteal (endocortical, intracortical, trabecular) (44). Each surface has a unique developmental pattern. The earlier growth in size than mass within a 

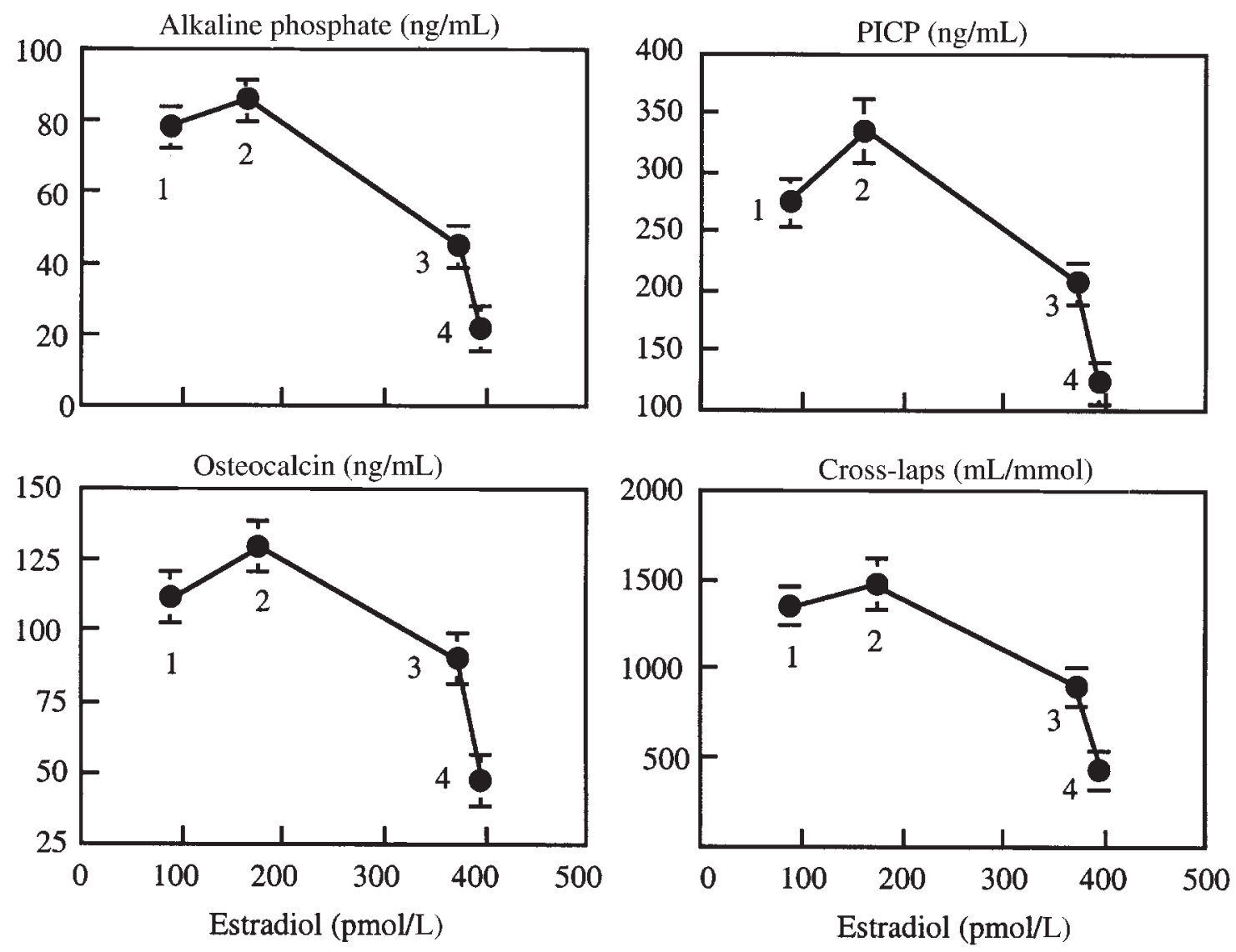

$\mathrm{n}=29,72,43,69$

Figure 7

Serum bone specific alkaline phosphatase, osteocalcin, collagen propeptide of type I collagen (PICP), and urinary type I C-telopeptide breakdown products (CrossLaps) versus estradiol. Numbers denote maturational stages; 1 (Tanner stage 1), 2 (includes Tanner stages $2-4$ ), 3 (1.0 \pm 0.1 years after menarche), and 4 ( $3.7 \pm 0.1$ years after menarche).

region, the differing tempo and direction of growth of the periosteal and endocortical surfaces, and the differing tempo of growth of the axial and appendicular skeleton suggests that each trait is regulated differently.

The heterogeneity in the growth of these traits provides the setting for the development of region-specific deficits in mass, size, or density should illness occur. That is, unlike in adulthood, the effect of exposure to a risk factor during growth depends on the maturational level of the region exposed (as well as the exposure "dose"). Thus, bone fragility is likely to have its origins established during growth. A better understanding of the pathogenesis of osteoporosis can be gained by identifying the genetic and environmental factors regulating the periosteal and endosteal surfaces of bone as the absolute and relative changes on these surfaces during growth and aging determine the size, mass, architecture, and strength of the skeleton (45).

\section{Acknowledgments}

The study was supported by the Dairy Research and Development Corporation of Australia. The authors thank the students and staff of the Ivanhoe Girls Gram- mar School and Sister Jan Edmonds for making this work possible.

1. Cooper, C., and Melton, L.J., III. 1996. Magnitude and impact of osteoporosis and fractures. In Osteoporosis. R. Marcus, D. Feldman, and J. Kelsey, editors. Academic Press Inc. San Diego, CA. 419-434.

2. Riggs, B.L., et al. 1981. Differential changes in bone mineral density of the appendicular and axial skeleton with aging: relationship to spinal osteoporosis. J. Clin. Invest. 67:328-335.

3. Riggs, B.L., and Melton, L.J., III. 1986. Involutional osteoporosis. N. Engl. J. Med. 314:1676-1686.

4. Eastell, R., et al. 1989. Unequal decrease in bone density of lumbar spine and ultradistal radius in Colles' and vertebral fracture syndromes. J. Clin. Invest. 83:168-174.

5. Mautalen, C., Vega, E., Ghiringhelli, G., and Fromm, G. 1990. Bone diminution of osteoporotic females at different sites. Calcif. Tissue Int. 46:217-221.

6. Seeman, E., et al. 1989. Reduced bone mass in daughters of women with osteoporosis. N. Engl. J. Med. 320:554-558.

7. Seeman, E., Hopper, J.L., Tsalamandris, C., and Formica, C. 1994. Bone density in daughters of women with hip fractures. J. Bone Miner. Res. 9:739-743.

8. Evans, R.A., et al. 1998. Bone mass is low in relatives of osteoporotic patients. Ann. Intern. Med. 109:870-873.

9. Ristevski, S., Yeung, S., Poon, C., Wark, J.W., and Ebeling, P. 1997. Osteopaenia is common in young first-degree male relatives of men with osteoporosis. Australian and New Zealand Bone and Mineral Society, Annual Scientific Meeting. Abstract book. Volume 7. Canberra, Australia. pp. 65.

10. Vega, E., et al. 1998. Bone mineral density and bone size in men with primary osteoporosis and vertebral fractures. Calcif. Tissue Int. 62:465-469. 
11. Gilsanz, V., et al. 1995. Gender differences in vertebral size in adults: biomechanical implications. J. Clin. Invest. 95:2332-2337.

12. Duan, Y., Parfitt, M., and Seeman, E. 1999. Vertebral bone mass, size and volumetric bone mineral density in premenopausal women, and postmenopausal women with and without spine fractures. J. Bone Miner. Res. In press.

13. Mazess, R.B. 1983. Non-invasive bone measurements in skeletal research. Volume 9. Academic Press. New York, NY. 277-343.

14. Robinson, R.A. 1960. Chemical analysis and electron microscopy of bone. In Bone as a tissue. K. Rodhal, J.T. Nicholson, and E.M. Brown, editors. McGraw-Hill Book Co. New York, NY. 186-250.

15. Parfitt, A.M. 1998. A structural approach to renal bone disease. J. Bone Miner. Res. 13:1213-1220.

16. Seeman, E. 1998. Growth in bone mass and size: are racial and gender differences in bone density more apparent than real? J. Clin. Endocrinol. Metab. 83:1-6.

17. Carter, D.R., Bouxsein, M.L., and Marcus, R. 1992. New approaches for interpreting projected bone densitometry data. J. Bone Miner. Res. 7:137-145.

18. Tabensky, A., Williams, J., Deluca, V., Brigante, E., and Seeman, E. 1996. Bone mass, areal and volumetric bone density are equally accurate, sensitive, and specific surrogates of the breaking strength of the vertebral body: an in vitro study. J. Bone Miner. Res. 11:1981-1988.

19. Garnero, P., et al. 1992. Measurement of serum osteocalcin with a human-specific two-site immunoradiometric assay. J. Bone Miner. Res. 7:1389-1397.

20. Garnero, P., and Delmas, P.D. 1993. Assessment of the serum levels of bone alkaline phosphatase with a new immunoradiometric assay in patients with metabolic bone disease. J. Clin. Endocrinol. Metab. 77:1046-1053.

21. Winterbottom, N., Vernon, S., Freeman, K., Dankoff, G., and Sevedin, S. 1993. A serum immunoassay for the C-terminal propeptide of type I collagen. J. Bone Miner. Res. 8(Suppl. 1):S341. (Abstr.)

22. Garnero, P., Gineyts, E., Riou, P.R., and Delmas, P.D. 1994. Assessment of bone resorption with a new marker of collagen degradation in patients with metabolic bone disease. J. Bone Miner. Res. 79:780-785.

23. Theintz, G., et al. 1992. Longitudinal monitoring of bone mass accumulation in healthy adolescents: evidence for a marked reduction after 16 years of age at the levels of the lumbar spine and femoral neck in female subjects. J. Clin. Endocrinol. Metab. 75:1060-1065.

24. Marubini, E. 1978. Mathematical handling of long-term longitudinal data. In Human growth: principles and prenatal growth. F. Falkner and J.M. Tanner, editors. Volume 1. 1st edition. Plenum Press. New York, NY, and London, United Kingdom. 209-225.

25. Fournier, P.-E., Rizzoli, R., Slosman, D.-O., Theintz, G., and Bonjour, J.P. 1997. Asynchrony between the rates of standing height gain and bone mass accumulation during puberty. Osteoporos. Int. 7:525-532.

26. Zhang, X.Z., Kalu, D.N., Erbas, B., Hopper, J.L., and Seeman, E. 1999. The effect of gonadectomy on bone size, mass and volumetric density in growing rats may be gender-, site-, and growth hormone-dependent. J.
Bone Miner. Res. 14:802-809.

27. Rosen, H.N., et al. 1995. Treatment with growth hormone and IGF-1 in growing rats increases bone mineral content but not bone mineral density. J. Bone Miner. Res. 10:1352-1358.

28. Bailey, D.A., Wedge, J.H., McCulloch, R.G., Martin, A.D., and Bernhardson, S.C. 1989. Epidemiology of fractures of the distal end of the radius in children as associated with growth. J. Bone Joint Surg. Am. 71:1225-1231.

29. Schibler, D., Brooks, C.G., Kind, H.P., Zachmann, M., and Prader, A. 1974. Growth and body proportions in 54 boys and men with Klinefelters syndrome. Helv. Paediatr. Acta. 29:325-333.

30. Lu, P.W., Cowell, C.T., Lloyd-Jones, S.A., Briody, J., and Howman-Giles, R. 1996. Volumetric bone mineral density in normal subjects, aged 5-27 years. J. Clin. Endocrinol. Metab. 81:1586-1590.

31. Warner, J., et al. 1998. Measured and predicted bone mineral content in healthy boys and girls aged 6-18 years: adjusted for size and puberty. Acta Paediatr. 87:244-249.

32. Gilsanz, V., et al. 1988. Vertebral bone density in children: effect of puberty. Radiology. 166:847-850.

33. Dunnill, M.S., Anderson, J.A., and Whitehead, R. 1967. Quantitative histological studies on age changes in bone. J. Pathol. Bacteriol. 94:275-291.

34. Gilsanz, V., et al. 1998. Differential effects of race on the axial and appendicular skeleton of children. J. Clin. Endocrinol. Metab. 83:1420-1427.

35. Katzman, D., Bachrach, L., Carter, D., and Marcus, R. 1991. Clinical and anthropometric correlates of bone mineral acquisition in healthy adolescent girls. J. Clin. Endocrinol. Metab. 73:1332-1339.

36. Glastre, C.C., et al. 1990. Measurement of bone mineral content of the lumbar spine by dual energy $\mathrm{x}$-ray absorptiometry in normal children: correlations with growth parameters. J. Clin. Endocrinol. Metab. 70:1330-1333.

37. Mazess, R.B., and Cameron, J.R. 1971. Skeletal growth in school children: maturation and bone mass. Am. J. Phys. Anthropol. 35:399-408.

38. Parfitt, A.M. 1997. Genetic effects on bone mass and turnover: relevance to black/white differences. J. Am. Coll. Nutr. 16:325-333.

39. Parfitt, A.M., Rauch, F., Travers, R., and Glorieux, F.H. 1999. A new model of cancellous bone growth. J. Bone Miner. Res. In press.

40. Garn, S. 1970. Nutritional perspectives. Charles C. Thomas. Springfield, IL. 3-120.

41. Smith, D.W., et al. 1976. Shifting linear growth during infancy: illustration of genetic factors in growth from fetal life through infancy. J. Pediatr. 89:225-230.

42. Seeman, E., and Hopper, J.L. 1997. Problems in the study of the genetics of osteoporosis. Osteoporos. Int. 7(Suppl. 3):s10-s16

43. Hopper, J.L., et al. 1998. Genetic, common environment, and individual specific components of variance for bone mineral density in 10- to 26year-old females: a twin study. Am. J. Epidemiol. 147:17-29.

44. Seeman, E., et al. 1982. The differential effects of endocrine dysfunction on the axial and appendicular skeleton. J. Clin. Invest. 69:1302-1309.

45. Seeman, E. 1997. From density to structure: growing up and growing old on the surfaces of bone. J. Bone Miner. Res. 12:1-13. 